\title{
Megasphaera elsdenii
}

National Cancer Institute

\section{Source}

National Cancer Institute. Megasphaera elsdenii. NCI Thesaurus. Code C86896.

A species of anaerobic, Gram negative, cocci shaped bacteria in the phylum Firmicutes.

This species is nonmotile, indole negative, produces acid from fructose, glucose and

maltose, does not reduce nitrate and is catalase negative. M. elsdenii is a commensal

organism of the intestinal tract and rarely has been shown to be an opportunistic pathogen in humans. 